\title{
Adaptación y estudio de las propiedades psicométricas del Cuestionario de consecuencias de consumo de alcohol en jóvenes, forma breve, en muestra de estudiantes universitarios españoles
}

\section{Adaptation and study of the psychometric properties of the Brief Young Adult Alcohol Consequences Questionnaire, in a Spanish university students sample}

Fecha de recepción: 21-01-2020

Fecha de aceptación: 03-06-2020
Begoña Espejo Tort

Departamento de Metodología de las Ciencias del

Comportamiento. Universitat de València

\section{resumen/ahstract:}

El objetivo de este trabajo es presentar la adaptación y estudio de las propiedades psicométricas del Cuestionario de consecuencias de consumo de alcohol en jóvenes, forma breve (B-YAACO) en una muestra de 323 estudiantes de Psicología españoles, de los cuales el $74.3 \%$ son mujeres. Se realizó una retro-traducción de los ítems siguiendo las normas de la International Test Commission (ITC), y un análisis factorial confirmatorio (AFC) con el programa Mplus mediante el método de extracción Weighted Least Squares Means and Variances adjusted (WLSMV) y la matriz de correlaciones policóricas. El AFC arrojó resultados satisfactorios sobre el modelo unidimensional, con muy buenos indicadores de ajuste. Todas las saturaciones factoriales presentaron valores adecuados y fueron estadísticamente significativas. La consistencia interna del instrumento es satisfactoria, así como los índices de homogeneidad. Asimismo, presenta buena evidencia de validez de criterio y validez diferencial. Con este instrumento se podrán evaluar las consecuencias derivadas del consumo de alcohol en jóvenes españoles con precisión, lo que permitirá proponer intervenciones de prevención de consumo de alcohol más ajustadas a las necesidades reales.

The aim of this work is to present the adaptation and study of the psychometric properties of the Brief Young Adult Alcohol Consequences Questionnaire (B-YAACQ) in a sample of 323 Spanish Psychology students, of which $74.3 \%$ are women. The items were back-translated following the International Test Commission (ITC) standards, and a confirmatory factor analysis (CFA) was conducted by means of the Mplus program, using the Weighted Least Squares Means and Variances adjusted (WLSMV) extraction method and the polychoric correlation matrix. Goodness of fit of the CFA showed satisfactory results on the one-dimensional model, and all the factor loadings were adequate and statistically significant. The internal consistency and the homogeneity indices of the instrument are good. The questionnaire also presents good evidence of criterion validity and differential validity. With this instrument, the consequences derived from alcohol consumption in Spanish young people could be evaluated with precision, which will allow to propose more adjusted interventions to the real needs to prevent alcohol consumption.

\section{palabras clave/keywords:}

AFC, Mplus, B-YAACQ, propiedades psicométricas

CFA, Mplus, B-YAACQ, psychometric properties 


\section{Introducción}

El patrón de consumo de alcohol que realizan actualmente los jóvenes se caracteriza por ingerir cantidades elevadas durante pocas horas y algunos días a la semana -binge drinking-, siendo además una realidad compartida por muchos países (Delegación del Gobierno para el Plan Nacional sobre Drogas, 2018; World Health Organization, 2019). Este consumo intensivo de alcohol lleva asociado la aparición de problemas biopsicosociales a corto, medio y largo plazo. En cuanto a los problemas a nivel biológico, se dispone de suficiente investigación realizada con adolescentes que evidencia los cambios funcionales y también estructurales que genera el consumo de alcohol en elevadas cantidades sobre cerebros que todavía se encuentran en período de maduración. Es notoria su influencia en el área pre-frontal encargada de las funciones ejecutivas, la atención, planificación, flexibilidad cognitiva, toma de decisiones y autocontrol, así como sobre el hipocampo encargado de la memoria y el aprendizaje. Ambas zonas son de las últimas en madurar y por tanto más vulnerables a sufrir cambios con el abuso de alcohol que se produce a partir de los 12 o 13 años, pudiendo incluso llegar a experimentar modificaciones irreversibles (Guerri y Pascual, 2019; Hagler et al., 2019; López-Caneda, Cadaveira y Campanella, 2018; Meruelo et al., 2019; Sullivan et al., 2019). Problemas de este tipo pueden ser las lagunas de memoria (Cox et al., 2019; McGovern, 2019; Wombacher, Matig, Sheff y Scott, 2019) y una mayor dificultad para pensar con claridad o para realizar correctamente tareas (Krieger, Young, Anthenien y Neighbors, 2018; McGovern, 2019; Naudé et al., 2019; Zamroziewicz et al., 2017), lo que puede dar lugar al fracaso y al absentismo escolar (Gakh, Coughenour, Assoumou y Vanderstelt, 2019; Niu, Jeong y Willoughby, 2020; Ribeiro, Fernandes y Macêdo, 2019).

Pero además de estas consecuencias a nivel biológico, esta forma de beber lleva asociada otro tipo de consecuencias entre las que se encuentran una mayor predisposición a progresar en la adicción (Naudé et al., 2019; Prince, Read y Colder, 2019; Tucker, Orlando y Ellickson, 2003) y la prevalencia de actividades de riesgo como por ejemplo participar en peleas o discusiones, conducir después de haber bebido o realizar conductas sexuales de riesgo (Cox et al., 2019; Krieger et al., 2018; McGovern, 2019; Niu et al., 2020).

Por otra parte, las consecuencias derivadas del consumo de alcohol actúan como moduladoras de las creencias que los jóvenes tienen respecto de su propio consumo. Concretamente, son las que determinan las creencias conductuales, ejerciendo su influencia sobre la actitud, la intención y la conducta de consumo intensivo de alcohol (DiBello, Miller y Carey, 2019; Kahler, Strong y Read, 2005; Pilatti y Godoy, 2010). A pesar de ello, la investigación sobre las consecuencias derivadas del consumo de alcohol entre población joven ha ocupado un lugar secundario, al considerarse menos graves dichas consecuencias que las que tiene el consumo de alcohol entre la población dependiente (Kahler et al., 2005).

No puede obviarse, además, que las consecuencias derivadas del consumo de alcohol se manifiestan de forma diferente en los jóvenes en comparación con los adultos (Kahler et al., 2005; Pilatti, Godoy y Brussino, 2010; Schulenberg \& Maggs, 2002; Verster, van Herwijnen, Olivier y Kahler, 2009), por lo que se estima necesario disponer de medidas de evaluación que den cuenta de la gama de problemas experimentados por estos jóvenes. Puesto que las consecuencias derivadas del consumo de alcohol no son las mismas entre los jóvenes 
que entre los adultos, Kahler y sus colaboradores se propusieron elaborar un instrumento que incluyese un conjunto de ítems indicativos de problemas menos severos, lo que podía ayudar a caracterizar de manera más precisa a los jóvenes consumidores. Hasta el momento solo se contaba con instrumentos incompletos para poder dar cuenta de la totalidad de consecuencias derivadas del consumo de alcohol entre jóvenes. Concretamente se evaluaba en algunos casos los problemas sociales y personales (Maddock, Laforge, Rossi y O’Hare, 2001; O'Hare, 1997) o el extremo más severo del espectro de consecuencias relacionadas con el consumo (Hurlbut y Sher, 1992), o no se tenía en cuenta la mayor o menor severidad de las consecuencias que pueden derivar del patrón de consumo (mayor número de consecuencias o más notorias en consumidores de mayor cantidad de alcohol o que consumen más días a la semana, etc.), o se manejaban instrumentos dirigidos a adolescentes que no daban cuenta de conductas como conducir tras haber consumido o mantener relaciones sexuales de riesgo, como por ejemplo el Rutgers Alcohol Problem Index (RAPI) (White y Labouvie, 1989) adaptado en España con posterioridad (López-Núñez, Fernández-Artamendi, Fernández-Hermida, Campillo-Álvarez y Secades-Villa, 2012). Todos estos aspectos trataron de superarse con el Young Adult Alcohol Consequences Questionnaire (YAACQ) (Read, Kahler, Strong y Colder, 2006), del que posteriormente se desarrolló una versión reducida (B-YAACQ), aunque curiosamente se publicara antes (Kahler et al., 2005). Los autores del YAACQ estudiaron sus propiedades psicométricas en una muestra de 340 estudiantes universitarios. Aplicaron el modelo de Rasch sobre los 48 ítems que configuraban dicho cuestionario para reducir su número, consiguiendo un grupo final de 24 ítems cuya estructura unidimensional fue confirmada posteriormente mediante un análisis de componentes principales. La consistencia interna (Alfa) presentó un valor de .83. Asimismo, obtuvieron buena evidencia de validez convergente con el RAPI $(r=.78)$ y comprobaron la no existencia de funcionamiento diferencial de los ítems (FDI) por sexo. En un estudio posterior (Kahler, Hustad, Barnett, Strong y Borsari, 2008) obtuvieron un buen indicador de fiabilidad test-retest con un intervalo de seis semanas entre ambas mediciones $(r=.70)$, así como buena validez de constructo al obtener una correlación estadísticamente significativa ( $r=.64)$ con la puntuación total en el Alcohol Use Disorders Identification Test (AUDIT) (Saunders, Aasland, Babor, de la Fuente, Juan y Grant, 1993).

El B-YAACQ ha sido adaptado en Alemania (Verster et al., 2009) y en Argentina (Pilatti et al., 2014). En el primer caso se utilizó una muestra de 667 estudiantes universitarios. Se constató la unidimensionalidad del cuestionario mediante un análisis de componentes principales, y la consistencia interna (Alfa) presentó un valor .82. Además, se obtuvieron evidencias de validez a partir de las correlaciones estadísticamente significativas entre la puntuación total en la escala de consecuencias y la puntuación total en el AUDIT $(r=.75)$, así como con diversas medidas relacionadas con el consumo de alcohol: número de días semanales de consumo de alcohol $(r=.49)$, número de bebidas alcohólicas consumidas por semana $(r=.63)$, número de días de consumo intensivo de alcohol (binge-drinking) a la semana $(r=.56)$, edad de inicio en el consumo de alcohol $(r=-.23)$, y tasa de alcohol en sangre de una noche de consumo $(r=.53)$. En la muestra argentina $(N=302$ estudiantes universitarios) también se aplicó el modelo de Rasch, pero no se estudió la unidimensiona- 
lidad mediante análisis de componentes principales ni factorial exploratorio. Tampoco se calculó el coeficiente de consistencia interna, aunque encontraron FDI en algunos ítems, y correlaciones estadísticamente significativas entre la puntuación total del cuestionario de consecuencias y diversas medidas relacionadas con el consumo: número de bebidas alcohólicas consumidas en un solo episodio $(r=.49)$, número de bebidas alcohólicas consumidas en dos horas seguidas $(r=.42)$ y número de borracheras de los últimos tres meses $(r=.59)$. Además, se calcularon diferencias entre grupos de manera que, quienes se emborrachaban y quienes realizaban consumo intensivo, presentaban un número significativamente mayor de consecuencias derivadas del mismo que quienes no realizaban este tipo de consumo.

El problema de estas dos adaptaciones, especialmente la argentina, es que no realizaron una retro-traducción como se indica en los estándares de la International Test Commission (ITC) (Muñiz, Elosúa y Hambleton, 2013; Muñiz, Elosúa, Padilla y Hambleton, 2016), sino que realizaron varias traducciones del inglés y compararon las mismas hasta llegar a un consenso. Esto no garantiza la equivalencia de significados entre palabras o expresiones de distintos países. En estos casos, la traducción literal puede hacer que ciertos ítems pierdan el sentido original de aquello que se pretende medir. Además, en el caso del español de Argentina hay palabras y expresiones que, incluso a pesar de tratarse de un idioma común con España, haya que adaptar o sustituir por otras.

Aun así, este instrumento ha obtenido evidencia de validez suficiente, al menos en su versión original, como para permitir evaluar con garantías las consecuencias que experimentan los jóvenes que realizan un consumo intensivo de alcohol. Por esta razón, el objetivo de este trabajo es adaptar y estudiar las propiedades psicométricas del B-YAACQ en muestra española. Disponer de un instrumento de estas características fiable y válido para población joven española, permitirá optimizar las intervenciones dirigidas a reducir el consumo intensivo de alcohol que llevan a cabo los jóvenes. Este conocimiento, junto con información detallada del patrón de consumo y los determinantes cognitivos que modulan la realización de esta conducta (expectativas asociadas al consumo, actitud frente al mismo, creencias, etc.), permitirá planificar de manera más eficiente intervenciones orientadas a reducir o controlar la conducta de consumo intensivo.

\section{Material y métodos}

\section{Participantes y procedimiento}

Se realizó una retro-traducción de los ítems del cuestionario siguiendo los estándares establecidos por la ITC (Muñiz et al., 2013; Muñiz et al., 2016). Una persona bilingüe tradujo los ítems del inglés original al español de España, y otra persona bilingüe diferente volvió a realizar la traducción al inglés. Estos ítems fueron enviados al propio Kahler, quien supervisó la equivalencia de ambas versiones en inglés. A partir de sus sugerencias en cuanto a equivalencia de significado, la redacción final de los ítems quedó como se presenta en el Anexo 1.

Se recogieron datos en papel de 338 estudiantes de Psicología de la Universitat de València en las propias aulas del centro en horario lectivo. La participación fue anónima y voluntaria, 
por lo que quien no quiso responder no participó en el estudio. Tras aceptar en la primera página el consentimiento informado, comenzaba el cuestionario.

Se preguntó a los participantes si habían consumido alcohol alguna vez o no, y quienes consumían alcohol configuraron la muestra de estudio $(N=325)$, ya que el objetivo era adaptar un cuestionario sobre las consecuencias que se habían experimentado después de consumir alcohol. De esta muestra de personas consumidoras de alcohol, el 74\% eran mujeres. El $65 \%$ eran estudiantes de primer curso y el resto de segundo curso. La media de edad de las personas participantes fue 18.71 años $(D T=1.12$ años, mínimo $=17$, máximo $=22)$, y la edad media de inicio en el consumo de alcohol fue de 14.43 años ( $D T=1.29$ años, mínimo $=11$, máximo $=19$ ).

\section{Variables e instrumentos de medida}

Se evaluó la frecuencia de consumo de alcohol preguntando por el número de días que habían consumido alcohol en cada uno de los seis meses anteriores al momento en el que se recogieron los datos. Las posibles respuestas iban desde ". Nunca" hasta "9. 9 días o más".

Se evaluó el consumo de alcohol actual en relación con el consumo cuando empezaban mediante la siguiente cuestión con tres opciones de respuesta: "Si comparas el consumo de alcohol que realizas ahora con el que realizabas al principio, puedes decir que...". Las opciones de respuesta fueron "1. Consumes menos cantidad", "2. Consumes cantidades parecidas" y "3. Consumes más cantidad”.

Se preguntó también si cada vez dedicaban más tiempo a planificar cómo conseguir bebida, a estar consumiendo, y a esperar que se pasaran los efectos de la bebida. Las tres cuestiones se respondían mediante una escala dicotómica (1. Sí, . No).

Puesto que estos jóvenes llevan tiempo consumiendo grandes cantidades de alcohol de manera compulsiva, se evaluó la posible aparición del "craving" mediante la siguiente cuestión: "Después de tomar unas copas tengo cada vez MÁS GANAS de beber”, que se respondía mediante una escala dicotómica (1. Sí, . No).

Como el consumo de alcohol se vincula a otros consumos, se evaluó también el consumo de marihuana preguntando directamente si se consumía marihuana o alguno de sus derivados, respondiendo mediante una escala dicotómica (1. Sí, . No).

Para evaluar el consumo de alcohol de riesgo se utilizó el Test de identificación de trastornos por consumo de alcohol (Alcohol Use Disorders Identification Test, AUDIT) (Saunders et al., 1993), adaptado posteriormente en España (Rubio, Bermejo, Caballero y SantoDomingo, 1998). El AUDIT consta de 10 ítems que evalúan la cantidad y la frecuencia de consumo (ítems 1-3), los posibles síntomas de dependencia (ítems 4-6) y los problemas relacionados con el consumo de alcohol (ítems 7-10). La puntuación final permite identificar la existencia de un posible consumo de riesgo en función de un punto de corte establecido. El formato de respuesta es tipo Likert, que va desde ". Nunca" hasta "4. 4 o más veces a la semana" para el ítem 1; desde ". Nunca" hasta "4. Todos o casi todos los días" para los ítems 2 al 8; y desde “. Nunca” hasta “4. Sí, en el último año” para los ítems 9 y 1. Los ocho primeros ítems se puntúan de 0 a 4 y los ítems 9 y 10 con 0,2 y 4 puntos respectivamente ( 3 
categorías). La puntuación global puede oscilar entre 0 y 4 . En la muestra de este estudio la consistencia interna (alfa de Cronbach) presenta un valor de .72.

Para evaluar el consumo actual de alcohol se pidió a los participantes que indicaran el número de consumiciones realizadas en una salida normal, y el número de consumiciones tomadas en un día de celebración o especial (cumpleaños, Nochevieja...). Esta medida ha sido ampliamente utilizada pero no es demasiado fiable porque no tiene en cuenta el tipo de consumición (fermentados y destilados tienen distinta graduación), por lo que se pidió además que indicaran en una tabla el consumo de alcohol que habitualmente realizan cuando salen los jueves, viernes y sábados, indicando hora por hora la bebida concreta consumida. Esto permitió obtener el número de horas que consumían de manera continuada y calcular los gramos de alcohol consumidos de una vez (durante al menos dos horas seguidas). Para ello se realizó la conversión del tipo de bebida y número de consumiciones a gramos de alcohol consumidos, calculando posteriormente la suma de gramos desde la hora de inicio hasta la hora de fin.

Por último, para evaluar las consecuencias del consumo de alcohol en jóvenes se utilizó la versión adaptada del Cuestionario de consecuencias de consumo de alcohol en jóvenes, forma abreviada (Brief Young Adult Alcohol Consequences Questionnaire, B-YAACQ) (Kahler et al., 2005). Este instrumento consta de 24 ítems que se responden mediante una escala dicotómica (1. Sí, . No). Permite obtener una puntuación total a partir de la suma de los ítems, indicando el número de consecuencias experimentadas por cada participante. Por tanto, la puntuación mínima posible sería 0 (ninguna consecuencia) y la máxima sería 24 (todas las consecuencias).

\section{Análisis de datos}

Para estudiar la estructura unifactorial del cuestionario se realizó un análisis factorial confirmatorio AFC mediante el programa Mplus 8.4 (Muthén y Muthén, 2017). Se empleó el método de estimación de mínimos cuadrados ponderados de medias y varianzas ajustadas (weighted least squares means and variance adjusted; WLSMV) para extraer la solución factorial. Este estimador se basa en la matriz de correlaciones policóricas, que se calcula cuando las variables son de respuesta ordinal o dicotómica (Asparouhov y Muthén, 2010; Brown, 2015) y ofrece estimaciones correctas de parámetros y de indicadores de ajuste (Flora y Curran, 2004). Además, no asume ningún tipo de distribución específica para las variables observadas (Sass, Schmitt y Marsh, 2014), que en este análisis son los ítems, por lo que no es necesario comprobar si la normalidad de la distribución de las respuestas a los mismos. La bondad de ajuste para cada modelo se evaluó utilizando el índice de ajuste comparativo (CFI), el índice de Tucker-Lewis (TLI) y el error de aproximación cuadrático medio (RMSEA). Con respecto a los índices CFI y TLI, valores mayores a .90 indican un ajuste adecuado y valores superiores a .95 un ajuste excelente (Bentler, 1990; Tucker y Lewis, 1973). Con respecto al RMSEA, valores menores a .08 indican un ajuste adecuado, y valores inferiores a .06 ajuste excelente (Steiger, 1990).

Para estudiar la fiabilidad de la escala se calculó el coeficiente Alfa de Cronbach, y se calcularon también los índices de homogeneidad corregidos de los ítems. 
Para obtener evidencias de validez de constructo se calcularon correlaciones entre la puntuación total en el cuestionario de consecuencias y la puntuación total en el AUDIT. Para obtener evidencias de validez de criterio, correlaciones entre la puntuación total en el cuestionario de consecuencias y para las variables relacionadas con los consumos de alcohol consideradas como criterio: edad de inicio en el consumo de alcohol, número de consumiciones en un día normal, número de consumiciones en un día de celebración, número de días que consumen alcohol en cada uno de los seis meses anteriores a la recogida de datos, y gramos totales de alcohol consumidos en jueves, viernes y sábado. Antes de calcular las correlaciones se comprobó la normalidad de la distribución de todas las variables mediante la prueba de Kolmogorov-Smirnov. Para las variables cuya distribución fue normal se calcularon las correlaciones de Pearson entre la puntuación total en el cuestionario de consecuencias y las variables relacionadas con los consumos de alcohol. Para las variables cuya distribución no fue normal, se calcularon correlaciones de Spearman. Se utilizó el método par a par (pairwise) de control de datos perdidos para no disminuir drásticamente el tamaño muestral. Se espera que todas las correlaciones sean positivas y estadísticamente significativas, excepto en el caso de la edad de inicio en el consumo de alcohol, que se espera negativa y estadísticamente significativa.

Asimismo, se comprobó la existencia de diferencias en el número de consecuencias (puntuación total en el cuestionario) en función de si cada vez dedican más tiempo o no a planificar cómo conseguir bebida, a estar consumiendo y a esperar que se pasen los efectos de la bebida. En caso de distribución normal de la puntuación total en el cuestionario de consecuencias se calcularon pruebas t. En el caso de no normalidad, pruebas no paramétricas U de Mann-Whitney. Los resultados esperados de estos contrastes son que quienes responden afirmativamente a estas tres cuestiones (cada vez dedico más tiempo a planificar cómo conseguir bebida, a estar consumiendo y a esperar que se pasen los efectos de la bebida), manifestarán mayor número de consecuencias derivadas de su consumo. Asimismo, se ha calculado otra prueba t para ver si hay diferencias en el número de consecuencias entre las personas que manifiestan tener cada vez más ganas de beber después de tomar unas copas (craving), y se espera que quienes contesten afirmativamente presenten mayor número de consecuencias. Por último, se ha calculado un análisis de varianza (ANOVA) con pruebas a posteriori de Scheffé (o un ANOVA no paramétrico de Kruskal-Wallis si la puntuación total en el cuestionario de consecuencias no se distribuye normalmente) para comprobar si existen diferencias en el número de consecuencias manifestadas en función de si actualmente consumen más, lo mismo o menos que cuando se iniciaron en el consumo de alcohol. Se espera que quienes consumen más en la actualidad presenten mayor número de consecuencias.

Todos estos análisis se realizaron con el paquete estadístico IBM SPSS, versión 26.

\section{Resultados}

El ajuste de los datos a un factor con los 24 ítems del cuestionario de consecuencias ofreció una solución no demasiado adecuada $(\mathrm{RMSEA}=.081$; TLI $=.895)$ debido a la baja variabilidad en los ítems 13 (he tenido sobrepeso a causa de la bebida) y 18 (he sentido que nece- 
sitaba beber después de levantarme (antes de desayunar)). En ambos casos solo un reducido número de participantes respondió afirmativamente a estas cuestiones $(1.9 \%(n=6)$ en ambos casos). Se trata de ítems que, probablemente, es difícil que se den en gente tan joven, especialmente el segundo de ellos. Tras eliminarlos del análisis la solución presentó ajuste excelente, ya que el RMSEA fue muy inferior a .06, y tanto el CFI como el TLI presentan valores muy superiores a .90 (ver Tabla 1). Las saturaciones factoriales oscilan entre .306 y .813 , y todas fueron estadísticamente significativas $(p<.001)$.

Tabla 1. Índices de ajuste del modelo unifactorial con 24 y con 22 ítems (sin ítems 13 y 18).

\begin{tabular}{lcccccc} 
& $\chi^{2}$ & gl & RMSEA & IC 90\% RMSEA & CFI & TLI \\
\hline Modelo unifactorial 24 items & $325.404^{* *}$ & 252 & .081 & {$[.060, .101]$} & .905 & .895 \\
\hline Modelo unifactorial 22 items & $262.664^{*}$ & 209 & .025 & {$[.011, .036]$} & .968 & .965 \\
\hline
\end{tabular}

Nota. $\mathrm{gl}$ = grados de libertad; RMSEA = root-mean-square error of approximation; IC = intervalo de confianza; $\mathrm{CFI}=$ comparative fit index; $\mathrm{TLI}=$ Tucker-Lewis index $* * p<.005 ; * p<.05$

La consistencia interna del cuestionario con 24 ítems presentó un valor de .805. Sin embargo, los índices de homogeneidad de dos ítems presentaban valores muy bajos (.135 y .058 para los ítems 13 y 18, respectivamente). Son los mismos ítems que, como se ha mencionado antes, apenas presentan variabilidad en las respuestas. Tras eliminarlos la consistencia interna subió a .807. Los índices de homogeneidad corregidos de los 22 ítems oscilaron entre .210 y .535 (ver Tabla 2).

La puntuación total en el cuestionario de consecuencias se calculó con los 22 ítems finales del cuestionario. Esta puntuación es la que se ha utilizado para los siguientes análisis de correlación y para las pruebas de contraste de hipótesis. Las pruebas de Kolmogorov-Smirnov realizadas previamente para las variables continuas indican que ninguna se distribuye normalmente $(p<.001$ en todos los casos), por lo que se calcularon los análisis no paramétricos.

La correlación de Spearman entre la puntuación total en el cuestionario de consecuencias y la puntuación total en el AUDIT fue estadísticamente significativa y en el sentido esperado $(r=.721, p<.001, N=323)$. Las correlaciones de Spearman calculadas para estudiar la validez de criterio se presentan en la Tabla 3.

Se puede observar que todas las correlaciones fueron estadísticamente significativas y en el sentido esperado, excepto para los gramos de alcohol consumidos los viernes. En este caso, no existe una relación estadísticamente significativa entre el número de consecuencias y la cantidad de alcohol consumida el viernes.

Las pruebas U de Mann-Whitney realizadas para comprobar las diferencias entre grupos se muestran en la Tabla 4. Se puede observar que todas son estadísticamente significativas y en el sentido esperado. Es decir, quienes manifiestan más consecuencias derivadas del 
Tabla 2. Alfa de Cronbach e índices de homogeneidad corregidos para la versión de 24 ítems y para la versión de 22 ítems ( $\sin$ ítems 13 y 18).

\begin{tabular}{|c|c|c|c|c|}
\hline & \multicolumn{2}{|c|}{ Cuestionario de 24 items } & \multicolumn{2}{|c|}{ Cuestionario de 22 ítems } \\
\hline & $\begin{array}{l}\text { Índice de homogeneidad } \\
\text { corregido }\end{array}$ & $\begin{array}{l}\text { Alfa de Cronbach si se } \\
\text { suprime el elemento }\end{array}$ & $\begin{array}{l}\text { Índice de homogeneidad } \\
\text { corregido }\end{array}$ & $\begin{array}{l}\text { Alfa de Cronbach } \\
\text { si se suprime el } \\
\text { elemento }\end{array}$ \\
\hline $\mathrm{Cl}$ & .295 & .801 & .296 & .803 \\
\hline $\mathrm{C} 2$ & .510 & .788 & .509 & .791 \\
\hline C3 & .322 & .800 & .323 & .802 \\
\hline $\mathrm{C} 4$ & .234 & .803 & .237 & .805 \\
\hline C5 & .215 & .807 & .221 & .809 \\
\hline C6 & .335 & .799 & .332 & .801 \\
\hline $\mathrm{C} 7$ & .355 & .798 & .354 & .800 \\
\hline C8 & .335 & .799 & .331 & .801 \\
\hline C9 & .445 & .793 & .447 & .795 \\
\hline $\mathrm{C} 10$ & .305 & .801 & .304 & .804 \\
\hline $\mathrm{C} 11$ & .326 & .799 & .325 & .801 \\
\hline $\mathrm{C} 12$ & .427 & .794 & .430 & .796 \\
\hline $\mathrm{C} 13$ & .136 & .805 & -- & -- \\
\hline $\mathrm{C} 14$ & .358 & .798 & .355 & .800 \\
\hline $\mathrm{C} 15$ & .480 & .790 & .479 & .793 \\
\hline $\mathrm{C} 16$ & .364 & .797 & .360 & .800 \\
\hline $\mathrm{C} 17$ & .378 & .797 & .373 & .799 \\
\hline $\mathrm{C} 18$ & .056 & .807 & -- & -- \\
\hline $\mathrm{C} 19$ & .206 & .804 & .210 & .806 \\
\hline $\mathrm{C} 20$ & .405 & .796 & .404 & .799 \\
\hline $\mathrm{C} 21$ & .540 & .789 & .535 & .791 \\
\hline $\mathrm{C} 22$ & .243 & .802 & .239 & .805 \\
\hline $\mathrm{C} 23$ & .484 & .792 & .483 & .794 \\
\hline $\mathrm{C} 24$ & .397 & .796 & .400 & .798 \\
\hline $\begin{array}{l}\text { Alfa de } \\
\text { Cronbach }\end{array}$ & & .805 & & .807 \\
\hline $\begin{array}{l}\text { Media de la } \\
\text { escala } \\
\text { Desviación }\end{array}$ & & 6.28 & & 6.25 \\
\hline $\begin{array}{l}\text { típica de la } \\
\text { escala }\end{array}$ & & 3.956 & & 3.931 \\
\hline
\end{tabular}


Tabla 3. Correlaciones de la puntuación total del cuestionario (sin ítems 13 y 18) con criterios.

\begin{tabular}{lccllc}
\hline & \multicolumn{3}{c}{$\begin{array}{c}\text { Puntuación } \\
\text { total cuestionario } \\
\text { consecuencias }\end{array}$} & & $\begin{array}{c}\text { Puntuación total } \\
\text { cuestionario } \\
\text { consecuencias }\end{array}$ \\
\hline Edad de inicio en el consumo & $\mathrm{r}$ & $-.261^{* *}$ & Días de consumo de alcohol & $\mathrm{r}$ & $.251^{* *}$ \\
de alcohol & $\mathrm{N}$ & 321 & hace 6 meses & $\mathrm{N}$ & 275 \\
Número de copas consumidas & $\mathrm{r}$ & $.418^{* *}$ & Número de días de consumo & $\mathrm{r}$ & $.359^{* *}$ \\
en día normal & $\mathrm{N}$ & 319 & de alcohol hace 5 meses & $\mathrm{N}$ & 304 \\
Número de copas consumidas & $\mathrm{r}$ & $.467^{* *}$ & Número de días de consumo & $\mathrm{r}$ & $.305^{* *}$ \\
en día de celebración & $\mathrm{N}$ & 311 & de alcohol hace 4 meses & $\mathrm{N}$ & 305 \\
Gramos de alcohol consumidos & $\mathrm{r}$ & $.267^{* *}$ & Número de días de consumo & $\mathrm{r}$ & $.245^{* *}$ \\
en jueves & $\mathrm{N}$ & 114 & de alcohol hace 3 meses & $\mathrm{N}$ & 276 \\
Gramos de alcohol consumidos & $\mathrm{r}$ & .065 & Número de días de consumo & $\mathrm{r}$ & $.266^{* *}$ \\
en viernes & $\mathrm{N}$ & 151 & de alcohol hace 2 meses & $\mathrm{N}$ & 236 \\
Gramos de alcohol consumidos & $\mathrm{r}$ & $.410^{* *}$ & Número de días de consumo & $\mathrm{r}$ & $.312^{* *}$ \\
en sábado & $\mathrm{N}$ & 255 & de alcohol hace 1 mes & $\mathrm{N}$ & 213 \\
\hline$* * p<.001$ & & & & &
\end{tabular}

consumo de alcohol son quienes dedican cada vez más tiempo a planificar cómo conseguir bebida, a estar consumiendo, a esperar que se pasen los efectos del alcohol, y a quienes manifiestan que después de tomar unas copas tienen cada vez más ganas de beber. Asimismo, las estimaciones del tamaño del efecto ( $\mathrm{d}$ de Cohen y r) permiten comprobar como en los tres primeros casos el efecto es medio y en el último caso (craving) el efecto de dicha variable sobre las consecuencias derivadas del consumo es grande.

Asimismo, la prueba de Kruskal-Wallis ofreció diferencias estadísticamente significativas $\left(H(2)=36.697, p<.001, \eta^{2}\right.$ parcial $\left.=.113\right)$ en el número de consecuencias manifestadas entre quienes consumen menos cantidad de alcohol que al principio $(M=5.47, D T=3.41)$, cantidades parecidas $(M=4.84, D T=3.55)$ y más cantidad de alcohol $(M=7.70, D T=$ 3.93). Las pruebas $U$ de Mann-Whitney realizadas entre los grupos revelan que quienes consumen actualmente más alcohol presentan un número de consecuencias significativamente mayor que quienes consumen lo mismo $(z=5.564, p<.001)$ y que quienes consumen menos que al principio $(z=4.145, p<.001)$. 
Tabla 4. Pruebas U de Mann-Whitney realizadas para estudiar la validez diferencial.

\begin{tabular}{|c|c|c|c|c|c|c|c|}
\hline & & $\mathrm{N}$ & Media & DT & $\begin{array}{l}\text { Valor d de } \\
\text { Cohen }\end{array}$ & $\mathrm{r}$ & $\mathrm{Z}$ \\
\hline Cada vez le dedico más tiempo a & Sí & 16 & 8.50 & 4.56 & \multirow{2}{*}{.55} & \multirow{2}{*}{.27} & \multirow{2}{*}{$-2.015^{*}$} \\
\hline planificar cómo conseguir bebida & No & 299 & 6.18 & 3.80 & & & \\
\hline Cada vez le dedico más tiempo a & Sí & 102 & 7.85 & 3.83 & \multirow{2}{*}{.60} & \multirow{2}{*}{.29} & \multirow{2}{*}{$-5.084 * *$} \\
\hline estar consumiendo & No & 216 & 5.58 & 3.70 & & & \\
\hline $\begin{array}{l}\text { Cada vez le dedico más tiempo a } \\
\text { esperar que me pasen los efectos de } \\
\text { la bebida }\end{array}$ & $\begin{array}{l}\text { Sí } \\
\text { No }\end{array}$ & $\begin{array}{l}64 \\
250\end{array}$ & $\begin{array}{l}7.33 \\
6.01\end{array}$ & $\begin{array}{l}3.22 \\
3.96\end{array}$ & .37 & .18 & $-3.096 * *$ \\
\hline $\begin{array}{l}\text { Después de tomar unas copas tengo } \\
\text { cada vez más ganas de beber }\end{array}$ & No & 233 & 5.55 & 3.69 & .75 & .35 & $-5.908 * *$ \\
\hline
\end{tabular}

Nota . DT $=$ desviación típica; $\mathrm{r}=$ tamaño del efecto

$* p<.05 ; * * p<.01$

\section{Discusión}

El objetivo de este trabajo ha sido realizar una adaptación y estudio de las propiedades psicométricas de la versión abreviada del Young Adult Alcohol Consequences Questionnaire (Kahler et al., 2005). Los resultados obtenidos con la muestra española confirman una estructura unidimensional del cuestionario, sin considerar los ítems 13 y 18 que apenas presentan respuestas afirmativas, lo que permite obtener una puntuación total en el cuestionario sin dichos ítems. Dicha puntuación reflejaría el número de consecuencias reportadas por los jóvenes consumidores de alcohol. Asimismo, el instrumento presenta una buena consistencia interna, similar a la obtenida en la versión original del mismo (Kahler et al., 2005) y en la versión alemana (Verster et al., 2009). Las correlaciones con variables relacionadas con el consumo de alcohol son prácticamente todas estadísticamente significativas y en el sentido esperado, como las obtenidas por los autores de la versión argentina del instrumento (Pilatti et al., 2014). Esto significa que el cuestionario de consecuencias presenta buena validez 
de constructo, ya que muestra correlaciones positivas y estadísticamente significativas con el AUDIT, que se utiliza también para evaluar las consecuencias del consumo de alcohol y distintos niveles de consumo de riesgo. Este resultado es similar a los obtenidos en las otras versiones del instrumento (Kahler et al., 2005; Pilatti et al., 2014; Verster et al., 2009). Por otra parte, la correlación con la edad de inicio en el consumo de alcohol es negativa y estadísticamente significativa, lo que quiere decir que cuanto antes se inicien los jóvenes en el consumo, mayor número de consecuencias van a referir. Este resultado es similar al obtenido en la versión argentina (Pilatti et al., 2014). En cuanto a las variables relacionadas con el consumo de alcohol (número de consumiciones en un día normal, número de consumiciones en un día de celebración, número de días que consumen alcohol en cada uno de los seis meses anteriores a la recogida de datos, y gramos totales de alcohol consumidos en jueves, viernes y sábado) las correlaciones son todas positivas y estadísticamente significativas con la puntuación total en la escala de consecuencias, excepto para los gramos de alcohol consumidos los viernes. Esto puede ser debido probablemente a que los jueves hay fiesta universitaria y se suele beber mucho. Por ello, los viernes salen menos, ya que además han tenido clase, y el sábado ya están en condiciones de volver a salir. De hecho, el número de estudiantes que consumen alcohol es mucho mayor jueves y sábado. En cualquier caso, los resultados indican que cuantos más días se consume alcohol al mes, y cuanto más alcohol se consume, mayor número de consecuencias derivadas del consumo se manifiesta. Estos resultados son similares a los obtenidos en la versión argentina (Pilatti et al., 2014), que incluían algunas de las variables que se han considerado en este trabajo relacionadas con la frecuencia de consumo, y a los resultados obtenidos en la versión alemana (Verster et al., 2009), que incluían variables de consumo, tanto de número de consumiciones como de tasa de alcohol en sangre.

Respecto a los ítems que presentan poca variabilidad en las respuestas, son ítems en los que pocos jóvenes manifiestan dichas consecuencias. Se trata del ítem 13 (he tenido sobrepeso a causa de la bebida) y el 18 (he sentido que necesitaba beber después de levantarme (antes de desayunar)). En la versión argentina ocurre algo similar con el ítem 18. Probablemente sea debido a que este ítem, en gente tan joven, es un indicador de dependencia que resulta demasiado severo en personas de esta edad. Si bien es posible que pueda ocurrir (de hecho 6 personas dicen que sí les ocurre), a estas edades tan jóvenes no es tan habitual. El caso del ítem 13 puede ser porque el sobrepeso se da cuando por cuestiones de ansiedad, además de beber (para olvidarse de los problemas, por ejemplo) se come demasiado. Quizá sea un indicador de otros problemas más allá del consumo de alcohol. Aun así, podrían darse estas consecuencias, especialmente la segunda, si se bebe demasiado, con demasiada frecuencia y desde demasiado joven. Por ello, aunque no formen parte de la estructura unidimensional del instrumento ni se utilicen para el cálculo de la puntuación total (que indicaría el número de consecuencias derivadas del consumo de alcohol), deberían incluirse como indicadores de problemas subyacentes más allá del consumo excesivo de alcohol, especialmente el ítem 18 que sería en sí mismo el primer indicador de dependencia. Esta información se podría complementar mediante el uso del AUDIT, diseñado especialmente para detectar consumos de riesgo y dependencia del alcohol. 
Por último, hay que informar de las limitaciones de este estudio. Por una parte, y en referencia a los ítems 13 y 18 que presentan pocas respuestas afirmativas, deberían estudiarse las propiedades psicométricas del instrumento en una muestra joven, pero de más edad. Si presentan un patrón de alta frecuencia de consumo de alcohol sostenido en el tiempo durante más años, es probable que los problemas de falta de respuestas afirmativas desaparezcan. Esto indicaría, por tanto, que dichos ítems son importantes para el total de la escala de consecuencias derivadas del consumo de alcohol, aunque quizá en otro tipo de población joven de más edad. Además, se ha utilizado únicamente muestra incidental de estudiantes universitarios, pero estos no son los únicos jóvenes que consumen alcohol. Por lo tanto, no se pueden generalizar los resultados a jóvenes de otras edades o bien que no estudien en la universidad. Debería ampliarse el estudio de las propiedades psicométricas de esta escala a otro tipo de jóvenes, cuyo patrón de consumo puede ser diferente. Por último, aunque esta es una versión en muestra española del cuestionario, puede no ser apropiada para otros países en los que se hable español, por lo que habrá que realizar adaptaciones y estudiar las propiedades psicométricas del instrumento en dichos países de habla hispana.

A pesar de las limitaciones del estudio, se puede decir que la versión adaptada del B-YAACQ (Kahler et al., 2005) sin los ítems 13 y 18 presenta buenas propiedades psicométricas en muestra española y ofrece una manera breve y eficiente de identificar los problemas derivados del consumo de alcohol en estudiantes universitarios.

\section{Referencias}

Asparouhov, T. y Muthén, B. (2010). Weighted least squares estimation with missing data. Mplus Technical Appendix, 2010, 1-1. Retrieved from http://www.statmodel.com/download/GstrucMissingRevision.pdf

Bentler, P. M. (1990). Comparative fit indexes in structural models. Psychological Bulletin, 107(2), 238-246. doi:1.1037/00332909.107.2.238

Brown, T. A. (2015). Confirmatory factor analysis for applied research Guilford publications.

Cox, M. J., Egan, K. L., Suerken, C. K., Reboussin, B. A., Song, E. Y., Wagoner, K. G. y Wolfson, M. (2019). Social and situational party characteristics associated with High Intensity alcohol use among youth and young adults. Alcoholism: Clinical and Experimental Research, 43(9), 1957-1966. doi:1.1111/acer.14143

Delegación del Gobierno para el Plan Nacional sobre Drogas. (2018). Estrategia Nacional sobre Drogas 2017-2024. Madrid: Secretaría General Técnica. Centro de Publicaciones.

DiBello, A. M., Miller, M. B. y Carey, K. B. (2019). Positive heavy drinking attitude mediates the association between college alcohol beliefs and alcohol-related outcomes. Addictive Behaviors, 88, 29-35. doi:1.1016/j.addbeh.2018.08.005

Flora, D. B. y Curran, P. J. (2004). An empirical evaluation of alternative methods of estimation for confirmatory factor analysis with ordinal data. Psychological Methods, 9(4), 466-491. doi:1.1037/1082-989X.9.4.466

Gakh, M., Coughenour, C., Assoumou, B. O. y Vanderstelt, M. (2019). The relationship between school absenteeism and substance use: An integrative literature review. Substance use \& Misuse, 1-12. doi:1.1080/10826084.2019.1686021

Guerri, C. y Pascual, M. (2019). In Aschner M., Costa L. G.(Eds.), Role of neuroinflammation in ethanol neurotoxicity Academic Press. doi:1.1016/bs.ant.2018.1.009

Hagler, D. J., Hatton, S., Cornejo, M. D., Makowski, C., Fair, D. A., Dick, A. S., . . Dale, A. M. (2019). Image processing and analysis methods for the adolescent brain cognitive development study doi:1.1016/j.neuroimage.2019.116091 
Hurlbut, S. C. y Sher, K. J. (1992). Assessing alcohol problems in college students. Journal of American College Health, 41(2), 49-58. doi:1.1080/07448481.1992.10392818

Kahler, C. W., Hustad, J., Barnett, N. P., Strong, D. R. y Borsari, B. (2008). Validation of the 30-day version of the brief young adult alcohol consequences questionnaire for use in longitudinal studies. Journal of Studies on Alcohol and Drugs, 69(4), 611-615. doi:1.15288/jsad.2008.69.611

Kahler, C. W., Strong, D. R. y Read, J. P. (2005). Toward efficient and comprehensive measurement of the alcohol problems continuum in college students: The brief young adult alcohol consequences questionnaire. Alcoholism: Clinical and Experimental Research, 29(7), 1180-1189. doi:1.1097/01.ALC.000017194.95813.A5

Krieger, H., Young, C. M., Anthenien, A. M. y Neighbors, C. (2018). The epidemiology of binge drinking among college-age individuals in the united states. Alcohol Research: Current Reviews, 39(1), 23-3.

López-Caneda, E., Cadaveira, F. y Campanella, S. (2018). Editorial: Binge drinking in the adolescent and young brain. Frontiers in Psychology, 9, 2724. doi:1.3389/fpsyg.2018.02724

López-Núñez, C., Fernández-Artamendi, S., Fernández-Hermida, J. R., Campillo-Álvarez, Á y Secades-Villa, R. (2012). Spanish adaptation and validation of the rutgers alcohol problem index (RAPI). International Journal of Clinical and Health Psychology, 12(2), 251.

Maddock, J. E., Laforge, R. G., Rossi, J. S. y O'Hare, T. (2001). The college alcohol problems scale doi:1.1016/S03064603(00)00116-7

McGovern, P. A. (2019). Novel risk factors for college student binge-drinking: A behavioral economics and satir growth odel perspective (Dissertation Abstracts International Section A: Humanities and Social Sciences). doi:1.17615/50p2-a323

Meruelo, A., Meruelo, A., Jacobus, J., Jacobus, J., Idy, E., Idy, E., ... Tapert, S. (2019). Early adolescent brain markers of late adolescent academic functioning. Brain Imaging and Behavior, 13(4), 945-952. doi:1.1007/s11682-018-9912-2

Muñiz, J., Elosúa, P. y Hambleton, R. K. (2013). Directrices para la traducción y adaptación de los tests: segunda edición. Psicothema, 25(2), 151-157. doi:1.7334/psicothema2013.24

Muñiz, J., Elosúa, P., Padilla, J. y Hambleton, R. K. (2016). Test adaptation standards for cross-lingual assessment. In C. S. Wells y M. Faulkner-Bond (Eds.), Educational measurement: From foundations to future (pp. 291-304, Chapter xvii, 494 Pages). New York, NY, US: Guilford Press, New York, NY.

Muthén, L. K. y Muthen, B. (2017). Mplus user's guide: Statistical analysis with latent variables, user's guide Muthén \& Muthén.

Naudé, G. P., Foster, R. N. S., Bartley, M., Martinetti, M. P., Ayers, L. O. y Reed, D. D. (2019). Predicting adverse consequences of alcohol consumption in underage college students using a novel fake ID purchase task. Experimental and Clinical Psychopharmacology, doi:1.1037/pha0000345

Niu, Z., Jeong, D. C. y Willoughby, J. F. (2020). Friends over doctors? the influences of source and perceived customization on college drinking. Health Communication, doi:1.1080/10410236.202.1712034

O'Hare, T. (1997). Measuring excessive alcohol use in college drinking contexts: The drinking context scale doi:1.1016/S0306-4603(96)00050-0

Pilatti, A. y Godoy, J. C. (2010). Construcción y valoración psicométrica del cuestionario de expectativas hacia el alcohol para adolescentes de argentina (CEA-A). Anales De Psicología, 26(2), 288.

Pilatti, A., Godoy, J. C. y Brussino, S. A. (2010). Construcción y valoración de las propiedades psicométricas del Cuestionario de Expectativas hacia el Alcohol para Niños de Argentina. Adicciones, 22(2), 113-124.

Pilatti, A., Read, J. P., Vera, B. d. V., Caneto, F., Garimaldi, J. A. y Kahler, C. W. (2014). The spanish version of the brief young adult alcohol consequences questionnaire (B-YAACQ): A rasch model analysis. Addictive Behaviors, 39(5), 842-847. doi:1.1016/j.addbeh.2014.01.026

Prince, M. A., Read, J. P. y Colder, C. R. (2019). Trajectories of college alcohol involvement and their associations with later alcohol use disorder symptoms. Prevention Science, 20(5), 741-752. doi:1.1007/s11121-018-0974-6

Read, J. P., Kahler, C. W., Strong, D. R. y Colder, C. R. (2006). Development and preliminary validation of the young adult alcohol consequences questionnaire. Journal of Studies on Alcohol, 67(1), 169-177. doi:1.15288/jsa.2006.67.169 
Ribeiro, F. R., Fernandes, B. R. y Macêdo, A. R. (2019). Consumption of alcohol and drugs and school absenteeism among high school students of public schools. Revista Brasileira De Enfermagem, 72(6), 1692-1698. doi:1.1590/0034-7167-20180828

Rubio, G. V., Bermejo, J. V., Caballero, M. S. y Santo-Domingo, J. C. (1998). Validation of the alcohol use disorders identification test (AUDIT) in primary care. Revista Clínica Española, 198(1), 11-14.

Saunders, J. B., Aasland, O. G., Babor, T. F., De la Fuente, Juan R y Grant, M. (1993). Development of the alcohol use disorders identification test (AUDIT): WHO collaborative project on early detection of persons with harmful alcohol consumption II. Addiction, 88(6), 791-804. doi:1.1111/j.1360-0443.1993.tb02093.x

Sass, D. A., Schmitt, T. A. y Marsh, H. W. (2014). Evaluating model fit with ordered categorical data within a measurement invariance framework: A comparison of estimators. Structural Equation Modeling: A Multidisciplinary Journal, 21(2), 167-18. doi:1.1080/10705511.2014.882658

Schulenberg, J. E. y Maggs, J. L. (2002). A developmental perspective on alcohol use and heavy drinking during adolescence and the transition to young adulthood. Journal of Studies on Alcohol, 54-7. doi:1.15288/jsas.2002.s14.54

Steiger, J. H. (1990). Structural model evaluation and modification: An interval estimation approach. Multivariate Behavioral Research, 25(2), 173-18. doi:1.1207/s15327906mbr2502_4

Sullivan, E. V., Brumback, T., Tapert, S. F., Brown, S. A., Baker, F. C., Colrain, I. M., . . Pfefferbaum, A. (2019). Disturbed cerebellar growth trajectories in adolescents who initiate alcohol drinking doi:1.1016/j.biopsych.2019.08.026

Tucker, J. S., Orlando, M. y Ellickson, P. L. (2003). Patterns and correlates of binge drinking trajectories from early adolescence to young adulthood. Health Psychology, 22(1), 79-87. doi:1.1037/0278-6133.22.1.79

Tucker, L. R. y Lewis, C. (1973). A reliability coefficient for maximum likelihood factor analysis. Psychometrika, 38(1), 1-1. doi:1.1007/BF02291170

Verster, J. C., van Herwijnen, J., Olivier, B. y Kahler, C. W. (2009). Validation of the dutch version of the brief young adult alcohol consequences questionnaire (B-YAACQ). Addictive Behaviors, 34(5), 411-414. doi:1.1016/j.addbeh.2007.09.013

White, H. R. y Labouvie, E. W. (1989). Towards the assessment of adolescent problem drinking. Journal of Studies on Alcohol, 5O(1), 30-37. doi:1.15288/jsa.1989.5.30

Wombacher, K., Matig, J. J., Sheff, S. E. y Scott, A. M. (2019). "It just kind of happens": College students' rationalizations for blackout drinking. Health Communication, 34(1), 1-1. doi:1.1080/10410236.2017.1384351

World Health Organization. (2019). Global status report on alcohol and health 2018. Geneva: World Health Organization.

Zamroziewicz, M., Raskin, S. A., Tennen, H., Austad, C. S., Wood, R. M., Fallahi, C. R., . . Pearlson, G. D. (2017). Effects of drinking patterns on prospective memory performance in college students. Neuropsychology, 31(2), 191-199. doi:1.1037/ neu0000313 


\section{Anexo 1.}

Indica si en el último año has sentido algunas de las cuestiones que se plantean a continuación:

C1.- He tenido resaca (dolor de cabeza, de estómago) la mañana después de haber estado bebiendo.

C2.- He asumido riesgos tontos cuando he estado bebiendo.

C3.- Cuando bebo en exceso no soy capaz de recordar lo ocurrido durante largos períodos de tiempo.

C4.- La calidad de mi trabajo o estudios han mermado por beber.

C5.- Me he sentido con menos energía o cansado por beber.

C6.- El beber me ha metido en relaciones sexuales de las que luego me he arrepentido.

C7.- A menudo he bebido en noches en las que había planeado no beber.

C8.- Mi apariencia física se ha visto perjudicada (dañada) por mi consumo de alcohol.

C9.- Cuando bebo digo o hago cosas vergonzosas.

C10.- Después de beber he sentido dolor de estómago o he vomitado.

C11.- No he ido a trabajar o he perdido clases porque estaba bebido/a, tenía resaca o me encontrada enfermo/a a causa de mi consumo

C12.- Mientras he estado bebiendo he hecho cosas impulsivas de las que me he arrepentido más tarde.

C13.- He tenido sobrepeso a causa de la bebida.

C14.- Me he despertado en un lugar inesperado tras beber mucho.

C15.- He pasado demasiado tiempo bebiendo.

C16.- Me he sentido mal conmigo mismo/a por mi forma de beber.

C17.- Mi consumo de alcohol me ha creado problemas con mi novia, con mi novio, con mi esposa/o, padres, o parientes cercanos.

C18.- He sentido que necesitaba beber tras levantarme (antes de desayunar).

C19.- He conducido mi coche cuando sabía que había bebido demasiado para conducir de manera segura.

C20.- He descuidado mis obligaciones con mi familia, trabajo o escuela por beber.

C21.- A menudo he tenido problemas para limitar la cantidad que bebo.

C22.- Me he desmayado por beber.

C23.- Me he vuelto muy grosero, desagradable, 0 insultante después de beber.

C24.- Me he dado cuenta de que necesitaba grandes cantidades de alcohol para notar algún efecto, o que no podría emborracharme con la misma cantidad que antes consumía. 